\title{
Spinal Cord Injury Paralysis: Synaptic Competitive-Learning and Rewiring Therapy Restores Locomotoricity
}

Venkata Krishnan $\mathbf{R}^{*}$

Department of Anatomy, Fiji School of Medicine, Suva, Fiji

\begin{abstract}
At this point in time there are only limited treatment options available for spinal cord injury (SCI) paralysis (e.g. implanted stimulation devices, stem cells). They are in clinical trials, complex, invasive, ill-affordable, and quality of outcome rather unpredictable. This author had presented two alternative treatment procedures based on over five decades of fundamental research on the functioning of the spinal cord motor system in 2009, 2013 and 2015 . These are non-invasive, functional, and recovery predictable. The first one uses low-dose Botulinum toxin to select paralyzed limb muscles. The second consists of transient partial denervation of the muscles. They both operate on similar principles of synaptic competitive-learning (SCL) and rewiring. They focus on the spinal motoneuron, its synaptic connectivity, and spinal cord reflexes. They are (i) induce competition among neuromuscular synapses, (ii) modify the motoneurons' soma sizes (Size principle) and thus their excitability-inhibitability, firing properties, (iii) resize the motor units of the paralyzed muscles, and (iv) reposition the synaptic memory weights impinging on the motoneurons. Animal model treated by the second procedure shows that even in complete cord injury, the residual networks of the isolated distal cord are capable of remarkable motor restoration (see videos). This paper now explains the precise mechanisms behind the restorative rewiring $(\mathrm{SCL})$ therapy.
\end{abstract}

Keywords: Botulinum toxin; Motor restoration; Neurorehabilitation; Size principle; Spinal Cord Injury (SCI); Spinal motoneuron; Synaptic Competitive-Learning (SCL); Synaptic memory weights

\section{Introduction}

As of now there are only limited numbers of treatment options available for spinal cord injury (SCI) paralysis. They are cord-implanted and/or exoskeletal stimulation devices and stem cells. Both are still in clinical trials. The former ones seem to have largely underestimated the self-reparative and restorative potentials inherent in the damaged spinal cord. Hence, they use the cord simply as conduit to pass electrical signals. Among the stem cells neurologists there is a broad consensus that a combination treatment strategy is possibly the best treatment approach fort SCI paralysis. Nevertheless, theirs seem to be invasive, complex, exorbitant, and quality of outcome rather unpredictable. For example, a particular stem cells procedure involves intra-cranial surgical extraction of olfactory bulb neurons. Secondly, neurotrophic growth factors had also been applied in these treatments. Several questions arise. Have the stem cells differentiated into functional neurons and synaptically reconnected with neurons /axons up and down the injury site? If so, what type/s of neurotransmitters they release? We are still unsure as to whether the stem cells, or the growth factors, or the decompression effect of the surgery either each one of them or all combined had contributed to the reported motor recovery. These questions remain yet to be answered [1]. In the meanwhile, this author had presented two alternative treatment procedures that are non-invasive, affordable, quality of recovery predictable, and can be improved upon by neuro-rehabilitation training. They are based on how the spinal cord motoneurons and their synapses learn and acquire locomotor skills by synaptic competitive-learning (SCL) and network wiring. Both the treatment procedures operate on similar reparative rewiring principles. This paper now describes in detail the precise neuronal mechanisms of the therapy and explains how in SCI the damaged cord circuitry can be rewired and significant motor recovery accomplished.

\section{Literature Review}

\section{Synapses competition during locomotor learning-matura- tion}

In the early 1970s it was found that in new born-neonatal animals each muscle fibre is innervated by as many as 3-4 motor axon terminals that come from different motoneurons (polyneuronal motor control). Then in the next several weeks most terminals withdraw and later each muscle fibre becomes controlled by one motor axon terminal. At that time, it was not clearly known what the cause of that initial multiple motor contacts and their subsequent regression was. Detailed studies later found there is actually competition between the motor axon terminals to gain control of that muscle fibre and the competitiveselection is directed by the participating motoneurons. This means, that initially motoneurons share each other's motor units and towards locomotor maturation the sizes of individual motor units become established. Jean-Pierre Changeux was the pioneer who expounded that the synapse competition is activity-dependent, learning-related, and to memory registering [2]. This is what is known as synaptic competitive-learning (SCL). We now know that SCL is not unique to the neuromuscular synapses. Motor learning-related SCL also operates in the cerebellar cortex Purkinje neurons, in the spinal cord motoneurons, Renshaw neurons, and cerebral primary motor cortex (M1) neurons $[2,3]$. Another striking example from human postnatal life is that, both sides' motor cortices project nearly equal numbers of corticospinal tract (CST) axons to each side of the spinal cord ventral horn neurons

*Corresponding author: Venkata Krishnan R, Department of Anatomy, Fiji School of Medicine, Suva, Fiji, Tel: 679 3233254; E-mail: krish_venk@yahoo.com

Received August 25, 2018; Accepted November 26, 2018; Published November 29, 2018

Citation: Krishnan RV (2018) Spinal Cord Injury Paralysis: Synaptic CompetitiveLearning and Rewiring Therapy Restores Locomotoricity. J Neurol Disord 6: 397. doi:10.4172/2329-6895.1000397

Copyright: @ 2018 Krishnan RV. This is an open-access article distributed under the terms of the Creative Commons Attribution License, which permits unrestricted use, distribution, and reproduction in any medium, provided the original author and source are credited. 
and compete to gain synaptic contacts. As the infant learns locomotor activity over 85 percent of axons from the contralateral motor cortex are selected and retained, while only around 15 percent CST axons retained from the ipsilateral motor cortex and locomotor maturity reached by around twelve years age [4]. This cumulative knowledge of SCL- wiring give us the clue and directive as to how we could now design new treatment procedures for SCI paralysis.

\section{The spinal motoneuron and its synapses}

Physiologists' perspective: The spinal alpha motoneuron is the largest of all brain neurons. The synapses that impinge on its soma, dendrites are conventionally classified as excitatory, inhibitory, and disinhibitory. Their potentials on the motoneuron membrane are graded, local, and non-propagating. It is at the axon-hillock that the action potential by the neuron is generated that propagates along the motor axon (Figure 1). The precise positioning of the synapse on the soma-dendrite is important: thus, an excitatory synapse that is positioned on a remote dendritic terminal will have very little excitatory effect on the neuron than if the same synapse is positioned on the axon-hillock. In the latter position that synapse will exert far reaching excitatory effect on that neuron [5].

Henneman's size principle of the motoneuron states that in a motoneuron its soma size is one of the most important determinants of its firing properties [6,7]. Excitability-inhibitability, membrane input resistance, duration of after hyper-polarization, axon conduction velocity is all soma size dependent. A large motoneuron innervates large numbers of muscle fibres (motor unit), and it is less readily excitable than the smaller ones (that innervate smaller motor units) that are more readily excitable. During muscle contraction, the smaller motoneurons are recruited (recruitment order of motor units) first and then in their increasing of order of soma size they further are recruited. When the muscle relaxes motoneurons are de-recruited in the reverse order of their sizes.

Cognitive systems perspective: An entirely new, but complementary dimension of understanding of the behaviour of motoneuron and its synapses comes from computational cognitive systems science. This is summarized: The synapses that impinge on the motoneuron represent its motor learned-experiences stored as memory weights. Together, all the weights (excitatory, inhibitory, and disinhibitory) constitute the motor memory map of that neuron. This map is a dynamic, malleable function map. Memory weights are not pre-formed at birth. Indeed, at birth most synapses are dummy weights. Weights are gradually loaded onto synapses during foetal, infant and throughout adult life as motor/locomotor skills are learnt and acquired. Furthermore, new weights are loaded and positioned closest to their functionally-related previously loaded weights. This is what is known as self-organizing and associative memory [8]. Indeed, the cerebral cortex somato-sensory (S1) and motor (M1) maps both are similar self-organizing, dynamic, malleable function maps. In the spinal motoneuron the weights on its function map are maintained in a continual excitation-inhibition balancing that regulates/ controls the normal discharge properties of that neuron. It should be noted that in SCI, the depletion of original weights is replaced by spontaneously grown compensatory, aberrant weights. These are neither competition-based, nor activity-dependent. The function maps on such motoneurons become disfigured, the excitation-inhibition balancing is severely disrupted. The neurons then fire in an erratic, un-controlled manner or fail to fire at all. New learning, memory registering, and recalls of previously learned motor skills into tasks execution are severely affected.

SCI paralysis: the clinical perspective in SCI, the spinal cord ventral horn neurons, cerebellar cortex, and thalamus become denervated at varying degrees of severity. In the next several weeks, the remaining intact inputs to these centres spontaneously sprout-out and reinnervate the denervated synaptic sites. Depending on the severity of injury the clinical picture presents as (i) spasticity/over-activity/paresis/paralysis across limb muscles, (ii) excitation-inhibition imbalance between synergists-antagonists, (iii) the motoneuron's firing properties are in disarray, (iv) orderly recruitment-derecruitment of motor units within and across muscles are impaired, (v) failure of adequate numbers of motor units activation presents as muscle weakness, (vi) abnormal cocontractions of synergists-antagonists muscles.

Taken together, the three above perspectives point out that in SCI paralysis reasonable motor restoration could be accomplished if:- (i) the sprouts of new aberrant connection weights are stalled, (ii) new weights are grown on the basis of activity-dependent, competitionbased (SCL) manner, (iii) and such weights be positioned by selforganizing and associative-memory registration on the motoneurons iv)this in turn will restore the excitation-inhibition balancing. The following sections describe how the SCL-rewiring therapy meets and full fills these requirements and restores locomotoricity.

\section{SCL-rewiring therapy to spinal cord paralysis- the back- ground}

Adult motoneuron soma size is modifiable: This author's findings are summarized:- motoneuron's soma size is not pre-determined at birth. Indeed, until late postnatal life the soma size is highly plastic, and closely related to motor/locomotor learning and maturation. While postnatal motoneurons send forth their axon terminals to multiinnervate the muscle fibres the soma sizes concurrently increase. Later as multi-innervation regresses the soma sizes decrease and stabilize ii) furthermore, adult motoneuron soma sizes are malleable by drugs, peripheral nerves surgery. For example, injecting Botulinum toxin in small dose to muscle induces sprouting of motoneuron axon terminals and many muscle fibres become multiply innervated. Meantime, the motoneurons' soma sizes increase. After several weeks when the effect of BoTx wears off, multi-innervation regresses and the soma sizes decrease and stabilize. Another method of inducing SCL is transient partial denervation the muscle. In this case, the intact motor axons sprout out and innervate the denervated muscle fibres. Their motor units' sizes as well as soma sizes increase. When the injured axons regenerate and arrive at their target muscle, competition (SCL) takes place between the intact, sprouted motor terminals and the regenerated terminals. It should be noted that while synapse competition takes place at the neuromuscular synapses a similar competition takes place on the motoneuronal synapses. Thus, both BoTx administration and partial denervation of muscle precisely re-enact SCL-wiring in the adult spinal cord motor system [3,9-11]. Computational modelling studies of the size principle have come out with far reaching findings: i) when a motoneuron increases its soma size it incorporates into its soma the functional properties of several of its interneurons, ii)the different sizes of the soma enable the member neurons of the pool for spectrally-timed discharging of their impulses [12] (Figure 2). The synapse competition and the fractionation of soma sizes enable the participating motoneurons for mutual error corrections in their discharge properties [13].

\section{In neonatal-new born age complete cord injury does not re- sult in paraplegia}

It is known since the 1950s that neonatal animals (rat, cat, dog) spinal cord complete transected could still reasonably recover to stand, 


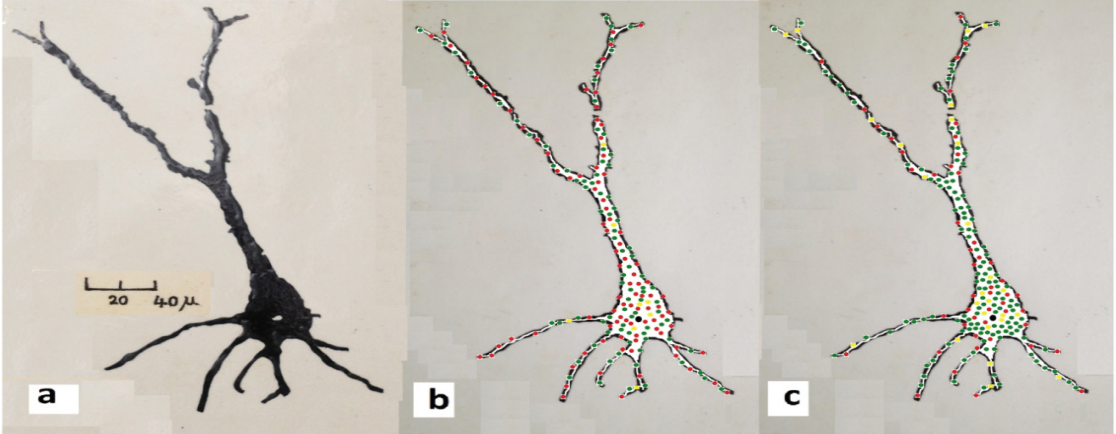

Figure 1: Memory weights on a motoneuron.

a) Alpha motoneuron of adult rat lumbar spinal cord (Golgi staining and camera lucida drawing by the author, 1978).

b) Note the synapses (green dots=excitatory; red=inhibitory; yellow=disinhibitory) are distributed in a mosaic pattern. Three principal factors determine the motoneuron's impulses firing properties: i) its soma size by Henneman in 1965 and 1979 [6,7] ii) where on the neuron i.e., dendritic terminal, dendritic stem, soma, the axon hillock, and which one of the three types of synapses is positioned there and, iii) the distances between these synapses (Granit, 1972) [5]. Cognitive systems sciences explain that the synapses represent the learned-experiences stored as motor memory weights. Throughout life the weights are maintained in a continual excitation-inhibition balancing.

c) In SCl, due to depletion of the original weights and their takeover by spontaneously grown aberrant weights the mosaic pattern of weights is severely disfigured. Excitation-inhibition goes off balancing. The motoneuron then fires in grossly erratic manner or fails to fire altogether.
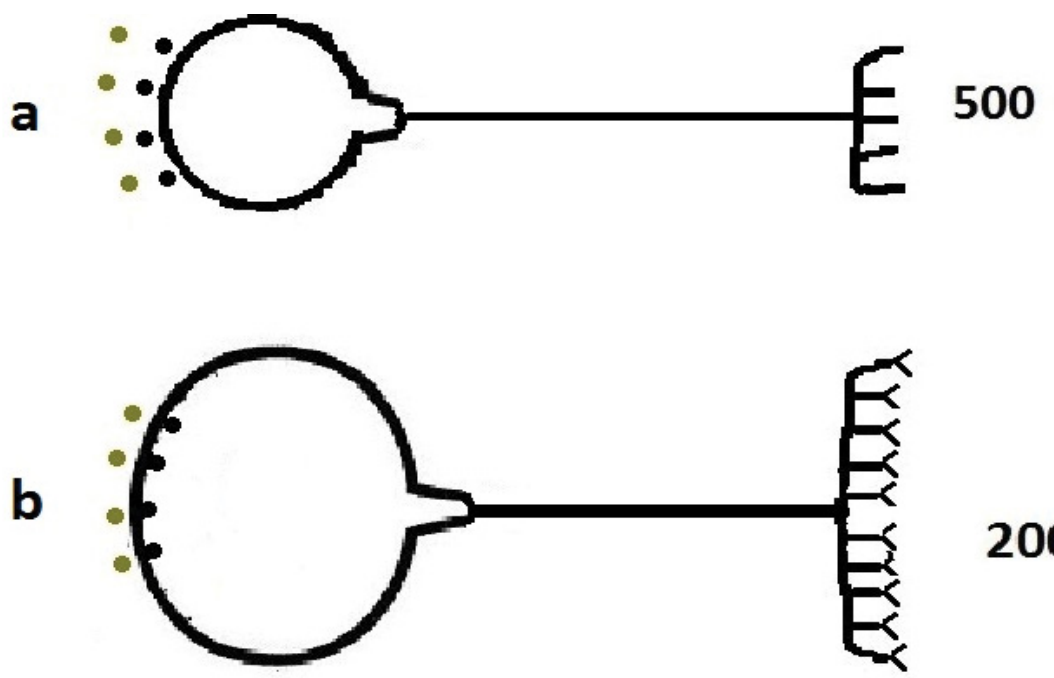

\section{0}

Figure 2: Plasticity of motoneuron soma size.

(a) and (b) are motoneurons of small and larger soma sizes that innervate motor units of 500 and 2000 muscle fibers respectively. Computational modeling of the size principle reveals that the larger motoneuron b) has incorporated into its soma the functional properties of several of its interneurons [11,12]. In early infant life the soma sizes as well as their motor units sizes are still in an indeterminate, plastic state as neuromuscular synapses competitions are still ongoing. It is at this crucial period that motoneurons, depending on their impending soma sizes incorporate/ or otherwise their interneurons. In other words, it is at this period that the adult pattern of discharge properties of motoneurons is determined. The SCL-rewiring therapy (by BoTx or transient partial denervation of muscles) precisely recreates the early infant life connectivity situation. The motoneurons' soma sizes become plastic and they stall the growth of aberrant weights. The residual weights become re-positioned based on activity-dependent, synapse competition and selection of connections. Excitation-inhibition balancing is restored. Functional motor restoration commences.

step, and climb slope as they grow into adults. For example, in rats cord transection in newborn and up to 18 days age does not result in paraplegia. They could manage themselves to support the body weight, stand, step, and climb a slope, lift the leg from falling into a grid hole. After 18 days however, a similar injury always results in permanent paralysis. This author found that this age difference in motor restoration in rats is due to that SCL- wiring mechanisms are still operative in the very young rats and SCL is already over beyond 18 days age. This finding opened the doors for designing the SCL-rewiring therapy to treat SCI paralysis in the adult. By reinstalling SCL mechanisms we can re-induce competition between neuromuscular synapses, modify the firing properties of motoneurons, re-position the connection weights, and resize the motor units. When very young SCI animals become capable of reasonable recovery into adults, why not in adult human?

\section{Animal model of complete SCI treated by transient partial denervation}

Amphibian model was preferred for the following reasons: a single nerve trunk (sciatic) from the lumbar spinal nerves 7-9 supplies all muscle groups of the entire hind limb. In a mammal this would mean surgery of four trunks viz. femoral, sciatic, obturator, and gluteal, 
coming from lumbar and sacral plexuses and a total of eight spinal nerves. Furthermore, long-term maintenance (over 16 weeks), and the eventual decubitus ulcers and, high mortality rates are almost nil in the amphibian as these animals dwell in their natural aquatic habitat. The neurosurgical procedure involves transient neurapraxia (controlled nerve crush) of the sciatic nerve trunk. This interrupts impulse conduction in about 20-30 percent of the sensory-motor axons. All the limb muscles become transiently partially denervated. Then, spontaneous sprouting of the intact motor axons follows within these muscles and they reinnervate the denervated fibres. When the interrupted motor axons regenerate and arrive (in the animal model six to eight weeks post-neurapraxia) into their muscles motor activitydependent synapse competition takes place between the sprouted and the regenerated motor terminals. Functionally appropriate connections are selected and stabilized while others are pruned. In the meantime, the motoneurons soma sizes become plastic and are resized. A similar synapse competition-selection takes place on their soma-dendritic surfaces. 6-10 weeks following the SCL-rewiring therapy swimming and stepping commenced in the treated animals and the restoration gained strength [11] (see videos). https://www.youtube.com/results?search_ query=krishnanspinal

\section{Discussion}

How do we know for certain that it is indeed the SCL-rewiring therapy that brought the motor restoration, and not due to regeneration across the injury site? First, none of the untreated control SCIc animals showed motor recovery. They remained complete paralyzed and indeed developed extensor-adductors spasticity and "scissoring" of the limbs. Second, in all the neurapraxia treated animals spasticity was absent. Instead, they regained the normal flexion position of the limbs. Third, the treated animals recovered swimming, body-weight support, and ground progression that persisted for several months of post-operative survival. After a complete injury the remaining inputs to the isolated cord segments are: - from muscle spindles (excitatory-stretch reflex), tendon organs (reciprocal inhibitory), joints capsule receptors, Renshaw neuron inputs (recurrent inhibitory), and from cutaneous receptors (excitatory). One might question: are these residuals, limited sensory inputs to the isolated cord enough and capable of driving locomotoricity in the limbs? The answer is: given the complete absence of all higher centres' inputs, reasonable and functional locomotoricity can still be restored just as what the neonatal SCI animals become capable of. More evidences are as follows- bipedal humanoid robotic modelling studies have shown that with a minimum set of sensorymotor neurons on each side, stepping, walking and obstacle avoidance can all be accomplished in a bipedal robot without higher centres control. Reflexes from the limbs and sensory inputs from foot contact alone are enough to maintain balance and gait with eight degrees of freedom in the limbs consisting of hip, knee and ankle joints [14]. An extensive study by Zehr and Stein found that in the adult human locomotion and gait maintenance muscular and cutaneous reflexes play dynamic, regulatory and modulatory roles. They further observe that in SCI these reflexes from the cord distal to the injury still retain their plasticity potentials to be exploited by neurorehabilitatory measures [15].

\section{Conclusion}

It is known that in human SCI over 70 percent of them are incomplete injuries. The animal model presented here shows that substantial functional motor recovery can be achieved even in severest injury. In other words, in incomplete SCI paralytics even far superior recovery results can be accomplished by the SCL-rewiring therapy.

\section{References}

1. Dalamagkas K, Tsintou M, Seifalian AM (2018) Stem cells for spinal cord injuries bearing translational potential. Neural Regen Res 13: 35-42.

2. Changeux JP, Danchin A (1976) Selective stabilization of developing synapses as a mechanism for the specification of neuronal networks. Nature (Lond) 264 : 705-712.

3. Krishnan RV (1983) A theory on the lability and stability of spinal motoneuron soma size and induction of synaptogenesis in the adult spinal cord. Int $\mathrm{J}$ Neurosci 21: 279-292.

4. Wolpaw JR, Kaas JH (2001) Taking sides: Corticospinal tract plasticity during development. Neurology 57: 1530-1531.

5. Granit R (1972) Mechanisms regulating the discharge properties of motoneurons. Nobel symposium. Liverpool University Press, UK.

6. Henneman E, Somjen G, Carpenter DO (1965) Functional significance of cell size in spinal motoneurons of different sizes. J Neurophysiol 28: 560-580.

7. Luscher, HR, Ruenzel P, Henneman E (1979) How the size of motoneurons determines their susceptibility to discharge. Nature 282: 859-861.

8. Kohonen T (1984) Self-organization and associative memory. Springer- Verlag: New York, USA.

9. Krishnan RV (2009) Botulinum toxin as a neuro-relearning drug tool in motor paralytic disorders. Curr Drug Ther 4: 101-105

10. Krishnan RV (2013) Restoring motor functions in spinal cord injury, hemiplegic cerebral palsy, and stroke by botulinum toxin-induced synaptic competitivelearning therapy. J Neurol Disord 1: 134.

11. Krishnan RV (2015) Spinal cord injury: Neurosurgical rewiring of neuromuscular and spinal cord circuitry restores locomotor functions. Open Access J Neurol Neurosurg 1: 1.

12. Grossberg S, Schmajuk NA (1989) Neural dynamics of adaptive timing and temporal discrimination during associative learning. Neural Networks 2: 79-102.

13. Stollberg J (1995) Synapse elimination, the size principle, and Hebbian synapses. J Neurobiol 26:273-282.

14. Paul C (2005) Sensorimotor control of biped locomotion. J Adapt Behav 13 67-80.

15. Zehr EP, Stein RB (1999) What functions do reflexes serve during human locomotion? Prog Neurobiol 58: 185-205. 